\title{
Synchronized GMTI Radar Collection Management in a Coalition Environment ${ }^{1}$
}

\author{
Andrew J. Newman \\ ALPHATECH, Inc. \\ Arlington, VA \\ Andrew.Newman@alphatech.com
}

\author{
John Emory \\ ALPHATECH, Inc. \\ Arlington, VA \\ John.Emory@alphatech.com
}

\author{
William H. Bennett \\ ALPHATECH, Inc. \\ Arlington, VA \\ Bill.Bennett@alphatech.com
}

\begin{abstract}
Surveillance of ground targets from airborne systems is increasingly of interest in tactical operations. Collection management synchronization can provide improved tactical data products for multi-platform operations. Synchronization of collection management is considered for coalition operations involving multiple airborne GMTI radar systems. An emerging synchronization collection management planning capability is evaluated for application to surveillance using GMTI radar systems. Several examples are provided which illustrate the capability and benefits of synchronized collection management.
\end{abstract}

Keywords: Collection management, resource management, multi-asset synchronization, coalition environment, GMTI/SAR simulation.

\section{Introduction}

Airborne Ground Moving Target Indicator (GMTI) radar has become an important asset for tactical surveillance of mobile ground targets. Tactical intelligence needs increasingly include requirements for detection and tracking of mobile ground targets. US and coalition forces now have available an increasing family of airborne GMTI radar collectors. To realize the intelligence requirement for target tracking some improvement in collection management will be needed. Coalition surveillance operations are particularly sensitive to deficiencies in collection synchronization. Synchronized GMTI radar collection planning can provide the improved coverage continuity needed for effective surveillance of mobile, ground targets.

In this paper, we advocate a new approach for collection management and synchronization of multiple GMTI radar airborne surveillance platforms for area surveillance. The approach is derived from emerging technology for optimization-based, mission planning and collection management developed as part of the DARPA Advanced ISR Management (AIM) program. We describe a tool for collection management synchronization called the MultiAsset Synchronizer and discuss its application to airborne radar surveillance collection planning. We highlight the emerging role of synchronized collection management in a coalition environment and illustrate application of collection planning capabilities for coalition operations.

The paper is organized as follows. The problem of collection management synchronization is formulated and capabilities of the Multi-Asset Synchronizer (MAS) are reviewed in Section 2. A brief overview of the role of synchronized GMTI radar collection management for coalition surveillance missions is provided in Section 3. Section 4 provides results from a series of simulation experiments on synchronized surveillance operations that involve plan construction using the MAS component and simulation of the mission execution. Section 5 provides details on the interface between the planning component and the simulator. Section 6 provides conclusions.

\section{Collection Synchronization}

\subsection{Need for Multi-Asset Synchronization}

The changing threat environment in which ISR operations occur and the continuing expansion of the role of ISR in military support present new challenges. The ISR confederation should provide tactically valuable information to the appropriate operational echelon within timelines consistent with the operational command tempo. Collection management must be responsive to requests for re-planning arising from ad hoc collection requirements and dynamic changes in threat posture. Current operational tempo is limited due to so-called "stove-piped" ISR management processes that have evolved for isolated sensor systems. ISR platforms have traditionally been deployed so as to enhance intrinsic sensor performance and not for contribution to combined information needs of command [1].

In response to these deficiencies, DARPA initiated the AIM program. Its objective is to "develop and transition capabilities that will allow the ISR confederation to operate in a time-compressed and cooperative collection capacity necessary for synergistic collections, time critical targeting, and dynamic battlefield awareness [2]." It is understood that coordination and synchronization of

\footnotetext{
${ }^{1}$ Work funded by AFRL/IF under contract F30602-01-C-0037 and DARPA/IXO under contract BT63-98-C-0068.
} 
multiple diverse ISR assets will enable significant gains in effectiveness and productivity (e.g., improved tracking and targeting accuracy) beyond that which is possible with stove-piped, single-asset management systems.

Given the complexity and growing size of the collection synchronization problem, it has been recognized that manual planning tools alone cannot meet the need for time-compressed planning cycles required to achieve satisfactory battlefield awareness. Automation techniques based on numerical optimization are needed that can rapidly generate synchronized collection plans that are optimized for total mission effectiveness. The key is to formulate a mathematical optimization problem that includes a meaningful, quantitative objective and captures the multiple operational constraints necessary to characterize the synchronization problem. This is discussed in the next section.

\subsection{MAS Collection Planning Problem}

In $[3,4,5,6]$ the multi-asset synchronization problem is formulated as a constrained, combinatorial optimization problem over a decision space of platform navigation courses of action and sensor-task-time assignments. The objective is to maximize the total information value collected while minimizing platform threat exposure.

The solution to the optimization problem is a collection plan including the following elements:

- a trajectory (i.e. flight path specified by space-time synchronization way-points) for each platform

- an assignment of collection tasks to each sensor

- a collection schedule for each sensor

The problem formulation accepts inputs as follows:

- Available assets: including a mix of airborne and spaceborne platforms with individually specified sensor configurations, operating region, on-station times, no fly zones, and threat risk tolerances. In addition, orbits for overhead platforms, and prescribed flight paths for certain airborne platforms, are specified as inputs.

- Target deck / tasking: including the set of collection tasks against which the available assets are to be assigned. Each task specifies a ground target location or area and collection requirements (e.g., time windows, revisit rates, image resolution, view angle, etc.). The task deck must be prioritized, i.e., each task has an assigned information value that has been determined according to military utility, commander's intent, etc.

- Enemy threat laydown : including the location and lethal radius of known enemy threats.
- Environment factors : including terrain elevation data and weather data for the operational area of interest.

- Mission data: the planning time horizon.

- Dynamic information requests and situation updates : including ad hoc tasks and changes to asset status and threat posture.

The collection plan must satisfy a variety of constraints on task-to-sensor allocations including:

- asset maneuverability

- sensor-to-target line-of-sight (LOS) intervisibility

- task availability time windows, deadlines, revisit periods, and continuity of area coverage

- sensor-to-target view angle/aspect

- airspace allocation and restrictions (flight operational areas, no-fly-zones, ingress/egress routes, prescribed orbits, and asset on/off-station times)

- sensor performance (e.g. radar target probability of detection, imaging sensor ground scene resolution, target geolocation precision)

The MAS problem is related to [6] a continuous space version of the Vehicle Routing Problem with Time Windows (VRPTW) (cf. [7]), a classical Operations Research problem known to be NP-hard. A rigorous mathematical representation requires joint consideration of continuous and discrete variables, linear and non-linear functions, and non-convex constraints. The number of variables and constraints becomes overwhelming quickly as the number of assets, number of tasks, and the planning horizon grows.

Dynamic replanning involves revising previously committed collection plans in response to dynamic requirements. The dynamic replanning problem is formulated by augmenting the MAS collection planning problem so as to penalize excessive schedules changes that produce marginal additional information value and to produce a feasible plan within a response time constraint.

\subsection{Synchronization Methodology}

An algorithmic approach was developed in $[3,4,5]$ that decomposes the MAS problem into two tractable subproblems: track (flight path) generation and sensor scheduling. The approach to the track generation subproblem uses an approximate dynamic programming methodology called Neuro-Dynamic Programming (NDP) $[8,9]$. An approximate "value-to-go" function for the optimization is computed based on a suitably defined multi-commodity network flow problem (NFP).

The sensor scheduling sub-problem is formulated as an assignment problem in which collection tasks are assigned 
to resource time intervals. Given platform routes, the tasks must be allocated to the different collection assets and a detailed sensor schedule must be generated for each asset. The approach generates a globally optimal assignment of tasks to assets consistent with collection requirements. A computationally efficient auction algorithm [10] is used during the assignment phase. For cases involving high sensor utilization, the solution to the assignment phase can often produce an infeasible schedule for one or more sensors. A second-phase scheduling algorithm was developed to compensate for this effect. It employs a dynamic programming approach to refine the initial schedule, by removing or relocating infeasible tasks and inserting unscheduled tasks when possible.

A dynamic replanning capability has also been developed. The algorithm is decomposed into three phases: a contingency recourse phase, a short-term recourse planning phase, and a long-term recourse planning phase. These phases differ primarily by the urgency of the decisions deadline, the types of decisions required, and the tolerance for perturbations to the existing plan. The replanning algorithms use techniques for time estimation and plan estimation to reduce the decision space and provide a robust approximate replanning solution while satisfying the decision deadline.

\subsection{A MAS Collection Management System}

The MAS software system provides an interface for the user to employ the models and algorithms described above to enable automated, optimization-based, operator-guided ISR collection planning and dynamic replanning. The system has been under development by ALPHATECH from 1997 through 2002 as part of the DARPA AIM program and related programs. It uses optimization technology and detailed models of ISR platforms and sensors to rapidly generate and dynamically maintain coordinated multi-INT collection plans for multiple ISR air-breathing and space-based assets. The MAS complements commander and personnel understanding of system capabilities and limitations by accepting user inputs, rapidly generating plans and replans on request, and enabling an improved understanding of the potential impact of asset reallocation and retasking decisions.

\section{Synchronized Coalition Operations}

Coalition surveillance operations are characterized by a diversity of platform and sensor capabilities operating against a common set of ground targets. Operational planning is shared according to coalition guidelines. Planning tools that facilitate rapid plan formulation, evaluation, and dissemination can contribute to operational effectiveness. Moreover, tools that can rapidly accept diverse sensor / platform capabilities and provide objective plan analysis can aid in acceptance and execution.
Optimized collection synchronization for multiple coalition airborne surveillance radars can provide improved surveillance data products for joint exploitation.

Improved wide-area surveillance can be achieved by synchronizing multi-platform sector GMTI coverage, revisit rates with SAR to achieve improved target detection quality commensurate with expected target behavior, and information needs. Synchronized collection can provided extended coverage continuity, enhanced revisit rate, multilateration capabilities for improved targeting, and crosscueing between GMTI and SAR modes for continuous coverage of mobile ground targets.

Growing recognition of these benefits within NATO has produced efforts to define the role of collection management in coalition operations and to incorporate procedures that permit cooperative, synergistic employment of multiple sensors. These capabilities will provide theatre Joint Task Force command with an enhanced situation awareness of threat ground activities.

Current coalition surveillance operations typically employ an ad hoc, decentralized sensor management architecture with an asset control stove-pipe for each platform. This architecture maintains a high degree of autonomy for the various command organizations and echelons, and consequent separation of control authority over individual assets. Cooperation is accomplished on an ad hoc basis. Requests for Information (RFI), Radar Service Requests (RSR), and asset control orders come from a variety of sources: on-board assets, ground-stations, command echelons, ISR manager, etc.

To promote synchronized collection management in a representative coalition environment, ALPHATECH has begun efforts toward :

- developing interfaces between the AIM/MAS component and coalition databases and communication networks that provide access to Air Tasking Order (ATO) / Airspace Control Order (ACO), prioritized RFI and RSR, live asset status updates, and weather data

- suggesting operating procedures that permit asset coordination and collaborative tasking

Coordination and synchronization of multiple coalition assets, as well as timely exploitation of GMTI and SAR radar data, are essential capabilities for effective dynamic tasking, sensor cross-cueing, and multi-platform cooperation. The tasking of coalition sensor resources should be flexible enough to enable the rapid re-targeting of GMTI and SAR, providing information products to commanders as required. To achieve these objectives, it will be necessary to enable rapid and responsive replanning. In the sequel we report on initial experiments 
in collection plan synchronization for continuous surveillance that illustrate the role of optimization for:

- rapidly generating and evaluating alternatives for asset allocation, tasking, and retasking, and resolving multiple trade-offs

- synchronizing multiple sensors to achieve improved tracking and targeting accuracy

- selecting "best" sensor to satisfy requests for information and cross-cues

- providing insight for trade-off / impact analysis, e.g., showing the value of allocating an additional asset or reassigning a given sensor

- ensuring satisfaction of collection task constraints (e.g. LOS intervisibility, etc.)

- planning and replanning platform trajectories that maximize collection and limit exposure to threats

- enforcing commander's intent, i.e., guidelines, constraints, and restrictions (e.g., airspace deconfliction, fixed/prescribed orbits, etc.)

- replanning in response to dynamic retasking

\section{Ground Surveillance Experiments}

To demonstrate the benefits of synchronized collection management, performed a series of experiments was performed using mission scenarios featuring continuous wide-area GMTI coverage of a single Named Area of Interest (NAI). The purpose of the experiments was to illustrate the potential of automated mission planning for synchronized collection management of multiple airborne GMTI radar systems in a coalition environment.

The experiments focused on synchronizing radar collections for both GMTI and SAR radar modes on multiple platforms. The platform suite included a notional, standoff asset (e.g. JSTARS) and a high altitude UAV (e.g. Global Hawk $(\mathrm{GH})$ ). The purpose of synchronization was to provide continuous GMTI coverage synchronized with collection of spot SAR imagery. An increasingly complex set of experiments was considered including additional high altitude manned and unmanned assets. Collection tasking requirements were also varied to illustrate the effectiveness of automated plan generation. Collection synchronization using cooperative teams including a standoff, manned surveillance asset and a high altitude UAV offers considerable flexibility for maintaining surveillance coverage since these systems offer complementary capabilities. Such an approach reflects a current US Air Force operational vision for ISR command and control, in which a manned "mother ship" equipped with multiple sensors operates in a "hen and chick" collaboration mode with a set of tethered UAV systems [11].

\subsection{Continuous GMTI Coverage}

This experiment demonstrates synchronization of JSTARS and GH UAV to maintain continuous GMTI WAS coverage during JSTARS service interruptions. Some interruptions of GMTI radar surveillance are unavoidable and occur during platform turns and when the ground target is shadowed from the platform by terrain. The loss of information during these blind times can be mitigated or eliminated through collaborative synchronization.

Scenario

Setting: $\quad$ Eglin FL, 60 min time horizon

Platforms: JSTARS (MTI/SAR), Global Hawk UAV (MTI/SAR, EO/IR)

GMTI Tasks: NAI 20x20 km - 60 min continuous WAS with $30 \mathrm{sec}$ update rate

SAR Tasks: none

Threats: none

Constraints: unconstrained view aspect

MAS Results

$\begin{array}{ll}\text { Coverage achieved: } & 59 \min (98.3 \%) \\ \text { JSTARS: } & 51 \mathrm{~min} \\ \text { GH UAV: } & 8 \mathrm{~min}\end{array}$

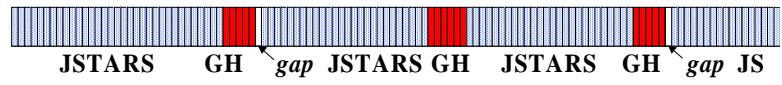

Figure 1: GMTI task allocation timeline

In this experiment, the MAS accepts a prescribed orbit for JSTARS and generates a GH UAV synchronized orbit to optimize the desired continuous coverage. The MAS schedules the GH UAV radar sensor to scan the NAI during JSTARS GMTI interruptions during platform turns. The task allocation timeline $(60 \mathrm{~min}$ divided into $30 \mathrm{sec}$ segments) and platform flight paths are shown in Figures 1 and 2. The synchronized plan achieves $98 \%$ coverage (two one-minute gaps) via collaborative synchronization.

\subsection{Aspect Constrained Surveillance}

This experiment is identical to the previous one, except that a view aspect constraint is imposed as part of the GMTI surveillance task requirement. Specifically, we stipulate that the sensor to target area line-of-sight be within 30 degrees of North or South. A constraint of this type can be used to enforce a minimum probability of detection for ground targets moving North or South. 


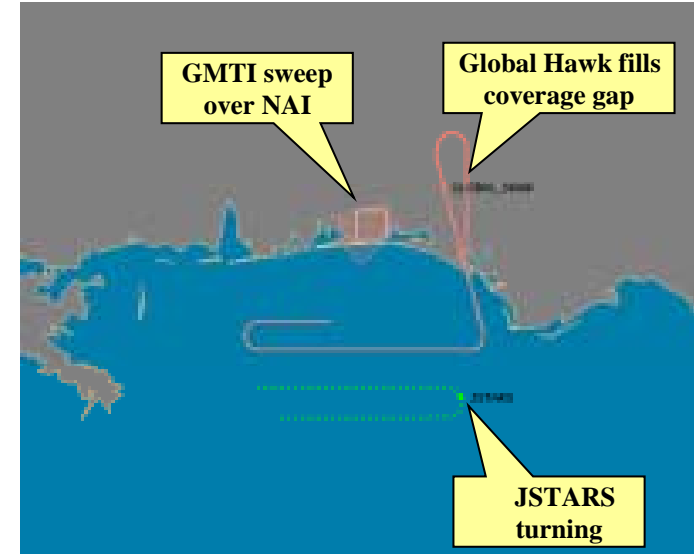

Figure 2: Collaboration for continuous coverage

$\underline{\text { Scenario }}$

Setting: $\quad$ Eglin FL, 60 min time horizon

Platforms: $\quad$ JSTARS (MTI/SAR), Global Hawk UAV (MTI/SAR, EO/IR)

GMTI Tasks: NAI $20 \times 20 \mathrm{~km}-60$ min continuous WAS with $30 \mathrm{sec}$ update rate

SAR Tasks: none

Threats: none

Constraints: N-S view aspect on NAI

MAS Results

$\begin{array}{ll}\text { Coverage achieved: } & 60 \mathrm{~min}(100 \%) \\ \text { JSTARS: } & 51 \mathrm{~min} \\ \text { GH UAV: } & 9 \mathrm{~min}\end{array}$

The MAS generates a flight path for the GH UAV such that it is in position to satisfy the desired view aspect while its radar is synchronized with JSTARS blind times. The task allocation timeline and platform flight paths are shown in Figures 3 and 4. The MAS achieves 100\% coverage via collaborative synchronization, a slight improvement over the result of the previous experiment. This example illustrates the near optimal property of MAS solutions, and shows how user guidance can improve performance.

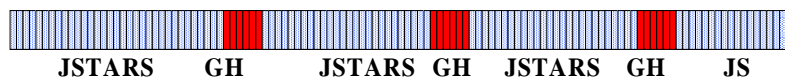

Figure 3: GMTI task allocation timeline

\subsection{Cross Aspect Surveillance}

This experiment requires that the GMTI surveillance be performed by two platforms simultaneously and from orthogonal view aspects. This requirement might arise to prevent MDV shadowing in regions of complicated terrain masking or to facilitate multi-lateration measurements on targets of interest.

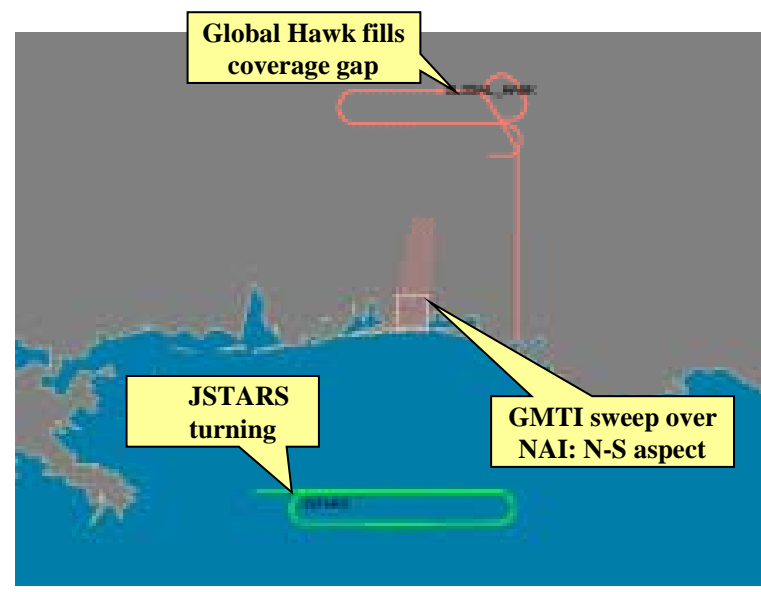

Figure 4: Collaboration to satisfy aspect constraint

$\underline{\text { Scenario }}$

Setting:

Platforms:

Eglin FL, 60 min time horizon

JSTARS (MTI/SAR), Global Hawk UAV (MTI/SAR, EO/IR)

GMTI Tasks: NAI 20x20 km - 60 min continuous WAS with $30 \mathrm{sec}$ update rate

SAR Tasks: none

Threats: none

Constraints: Simultaneous N-S and E-W view aspects

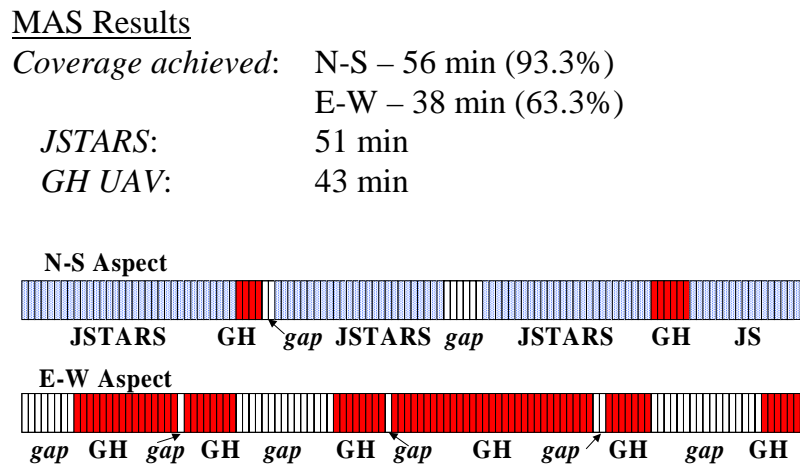

Figure 5: GMTI task allocation timeline

The MAS generates a flight path for the GH UAV in order to provide complementary orthogonal coverage as well as to compensate during JSTARS blind times where possible. The task allocation timeline and platform flight paths are shown in Figures 5 and 6. The MAS achieves 93\% NorthSouth coverage and $63 \%$ East-West coverage via collaborative synchronization. Besides providing surveillance from the East-West aspect, GH UAV also covers 5 of the 9 minutes during which JSTARS is turning. 


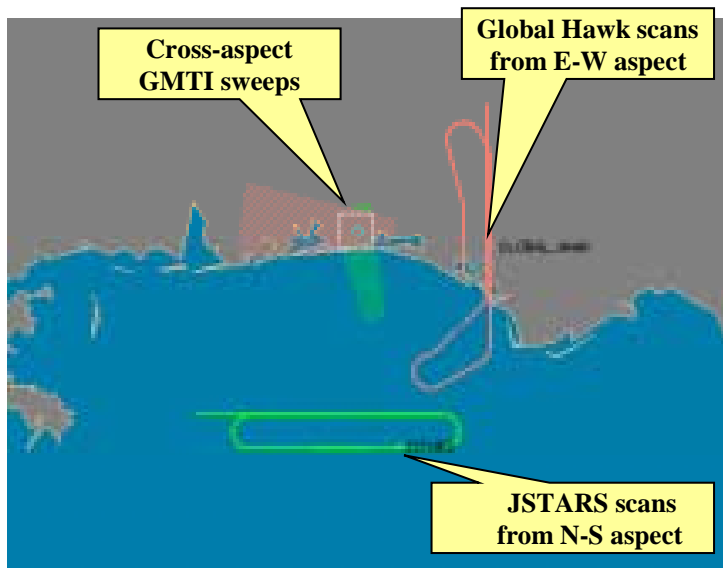

Figure 6: Collaboration to satisfy cross-aspect surveillance

\subsection{Priority Spot SAR}

This experiment requires that spot SAR imagery be collected at periodic intervals to complement GMTI WAS surveillance. We specify a spot target within the NAI and require collection of 0.3 meter resolution SAR imagery at 20 minute intervals starting 10 minutes into the plan. The GMTI surveillance task remains as above, with a requirement for North-South view aspect.

\section{$\underline{\text { Scenario }}$}

Setting: $\quad$ Eglin FL, 60 min time horizon

Platforms: $\quad$ JSTARS (MTI/SAR), Global Hawk UAV (MTI/SAR, EO/IR)

GMTI Tasks: NAI 20x20 km - $1 \mathrm{hr}$ continuous WAS with $30 \mathrm{sec}$ update rate

SAR Tasks: $\quad$ Sequence of 3 spot SAR at $20 \mathrm{~min}$ intervals

Threats: none

Constraints: N-S view aspect on NAI

\section{MAS Results}

$\begin{array}{lc}\text { GMTI coverage achieved: } & 60 \text { min }(100 \%) \\ \text { JSTARS: } & 51 \text { minutes } \\ \text { GH UAV: } & 9 \text { minutes } \\ \text { Spot SAR achieved: } & 3(100 \%)\end{array}$

The MAS generates a flight path for the GH UAV in order to collect the spot SAR while also providing GMTI coverage during JSTARS blind times where possible. For this problem, MAS is able to schedule all three spot SAR tasks as well as provide $100 \%$ GMTI coverage. The task allocation timeline for GMTI is identical to that in Figure 3.

Figures 7, 8, and 9 show a sequence of task allocations at consecutive simulated time steps (30 sec step size) as scheduled by MAS. The sequence illustrates the power of multi-asset synchronization. The flight paths and tasking schedules for JSTARS and GH UAV are synchronized such that (1) the UAV is positioned to collect a SAR image while JSTARS provides GMTI surveillance, followed immediately by (2) the UAV switches to GMTI WAS mode to compensate during the JSTARS platform turn.

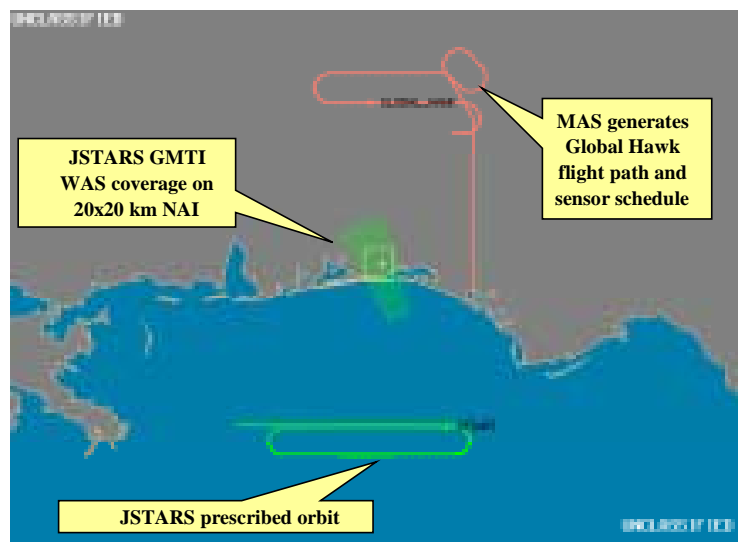

Figure 7: JSTARS GMTI WAS coverage

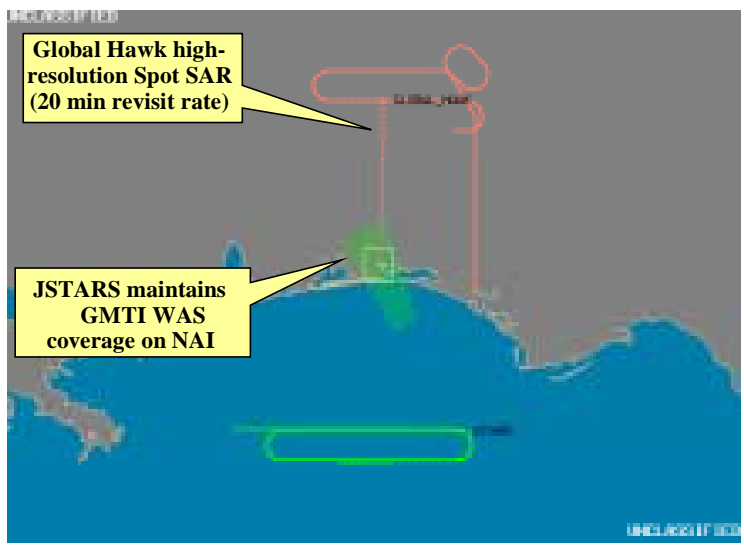

Figure 8: Synchronized GH UAV spot SAR

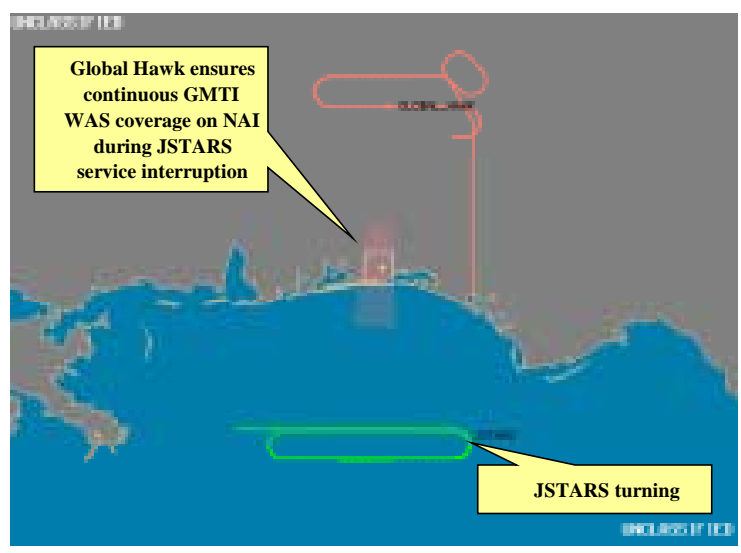

Figure 9: GH UAV covers JSTARS blind spot 


\subsection{Multi-Surveillance Target Mission}

In this experiment we increase the complexity by scaling up the number and diversity of tasks and available assets and by including enemy threats. The scenario features four platforms (JSTARS, GH UAV, U2, and Predator UAV), three GMTI surveillance NAIs of varying size $(100,20$, and $10 \mathrm{~km}$ square) and varying desired update rate $(30-60 \mathrm{sec})$, and a distribution of imagery tasks (11 spot SAR and $36 \mathrm{EO}$ ) and enemy threats over the theatre of interest.

\section{$\underline{\text { Scenario }}$ \\ Setting: \\ Platforms: \\ Eglin FL, 60 min time horizon \\ JSTARS (MTI/SAR), GH UAV \\ (MTI/SAR, EO/IR), U2 (MTI/SAR), Pred \\ (SAR, EO/IR) \\ GMTI Tasks: NAI \#1 100x100 km - $1 \mathrm{hr}$ continuous \\ WAS with $60 \mathrm{sec}$ update rate \\ NAI \#2 20x20 km - 10 min continuous \\ with $30 \mathrm{sec}$ update rate \\ NAI \#3 10x10 km - 10 min continuous \\ with $30 \mathrm{sec}$ update rate \\ SAR Tasks: 11 spot imagery \\ EO Tasks: $\quad 36$ spot imagery \\ Threats: $\quad 2$ SA-x \\ Constraints: $\quad \mathrm{N}-\mathrm{S}$ view aspect on NAI \#1}

MAS Results

GMTI Coverage Achieved

\begin{tabular}{|lllll|}
\hline NAI & Coverage & JSTARS & GH UAV & U2 \\
1 & $97 \%$ & $43 \mathrm{~min}$ & $2 \mathrm{~min}$ & $13 \mathrm{~min}$ \\
2 & $100 \%$ & $8 \mathrm{~min}$ & $1.5 \mathrm{~min}$ & $0.5 \mathrm{~min}$ \\
3 & $100 \%$ & $10 \mathrm{~min}$ & & \\
\hline
\end{tabular}

Spot SAR achieved: $92 \%$ - GH 14, Pred 19

Optical achieved: $\quad$ 100\% - GH 6, U2 2, Pred 3

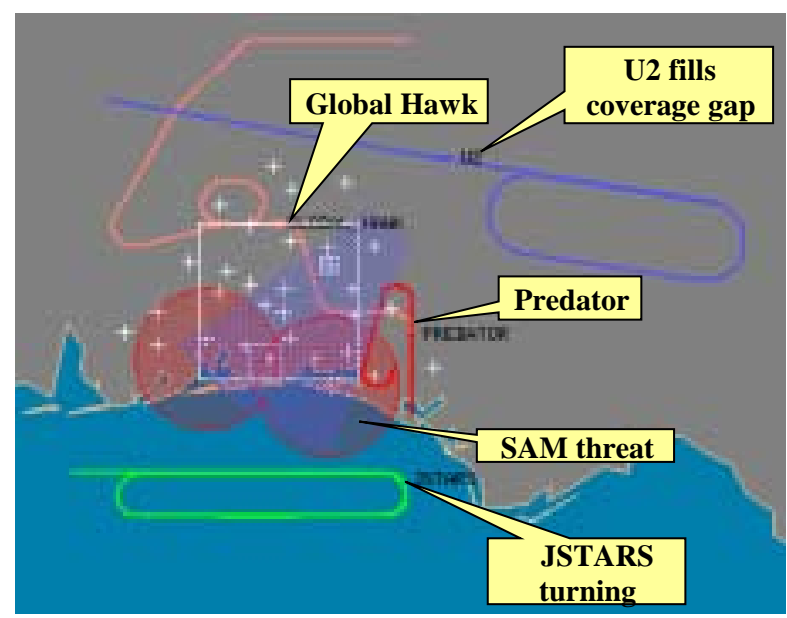

Figure 10: Multi-asset synchronization to maximize GMTI surveillance coverage and imagery collection
As before, MAS accepts a prescribed orbit for JSTARS and generates a flight path for the other available platforms (Figure 10). The MAS schedules a collaboration of JSTARS, GH UAV, and U2 to provide continuous GMTI coverage on the three NAIs. There are two one-minute gaps in coverage on NAI \#1 (representing 3\% of total requested) that MAS was not able to fill. In addition, MAS schedules a collaboration of GH UAV, U2, and Predator UAV to collect 44 of 47 imagery tasks requested.

\section{Simulator Interface}

AFRL/IF has developed UAVSIM as a stand-alone UAV simulator [12], referred to as UAVSIM, that simulates platform/sensor operations including GMTI/SAR radar operation. The simulator accepts a stream of DIS truth entity PDUs (ground truth) and employs a radar detection model to produce a realistic stream of radar detection reports. The simulator supports generic models for a number of platforms and sensors including Global Hawk SEP, Predator, and U2 ASARS (see [12] for details).

ALPHATECH developed a software interface to provide interoperability between MAS and UAVSIM. This interface translates the multi-asset collection plan generated by MAS into the input file formats required to drive a UAVSIM GMTI/SAR mission simulation. This interface will be used to support coalition exercises and experiments, and may be viewed as an early step toward interfacing synchronized collection management capabilities with operational asset management systems.

The interface architecture developed for this project is illustrated in Figure 11. This includes an upstream component, called the mission interface, that accepts a multi-asset GMTI/SAR mission description consistent with UAVSIM and produces input data files that drive the MAS planning algorithms. The MAS then sends optimized multi-asset collection plans to a downstream component called the MAS-UAVSIM interface, which drives the flight and sensor simulation routines in UAVSIM. UAVSIM then simulates the mission, displaying the platform flight and sensor detection stream (GMTI dots/blobs) superimposed on a cartographic representation of the area.

Figure 12 shows a snapshot from a UAVSIM simulation of a collection plan generated by MAS corresponding to the cross-aspect surveillance experiment (see Section 4.3). 


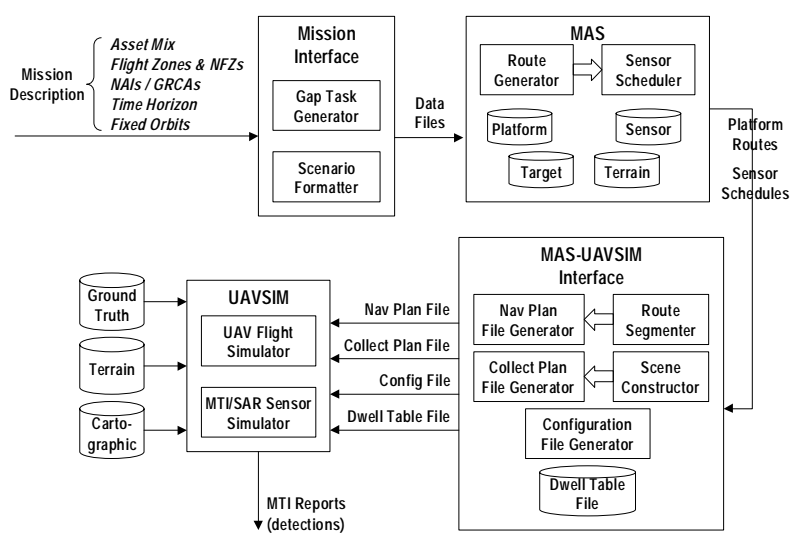

Figure 11: MAS-UAVSIM interface architecture

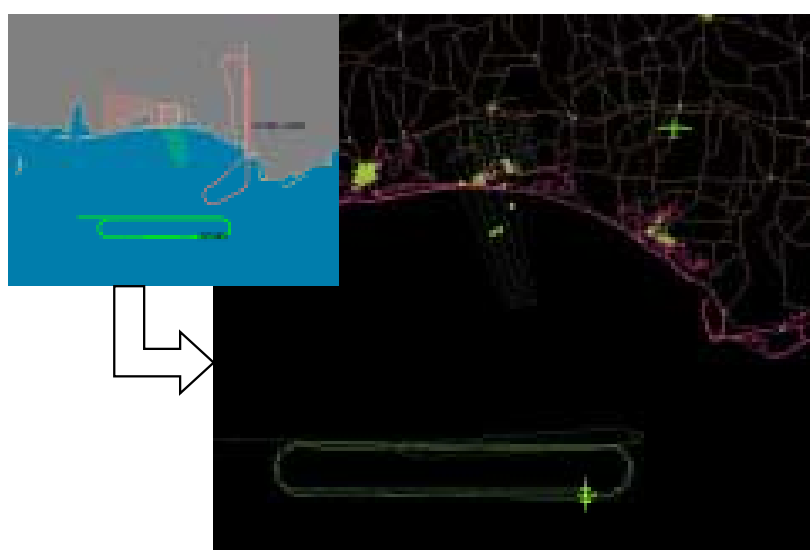

Figure 12: UAVSIM simulates MAS collection plan

\section{Conclusions}

We have demonstrated an application of collection management synchronization to multiple platform radar surveillance of ground targets. An interface between the AIM/MAS planning tool and the UAVSIM simulator was developed to support this analysis. Experimental results were obtained that demonstrate improved surveillance coverage and ISR performance achieved through coordination and synchronization of multiple airborne platforms. This capability can enable improved coalition operations. In a related effort [13] we have explored improvement in track fusion from collaborating platform GMTI coverage for challenging tactical surveillance problems. Future plans are to evaluate track improvement from collection synchronization and centralized track fusion.

\section{References}

[1] "Advanced ISR Management, PAD" Defense Advanced Research Projects Agency (DARPA), Information Systems Office (ISO), 1999.
[2] “Advanced ISR Management, Proposer's Information Package," DARPA, ISO. Version 0.1, July 23, 1997.

[3] C. J. Donohue, J. A. Emory, O. P. Kreidl, and C. T. Lawrence, "Advanced Collection Management, Final Report," ALPHATECH Technical Report TR-939, ALPHATECH, Inc., August 1999.

[4] C. J. Donohue, J. A. Emory, J. P. Marsh, and C. L. Morefield, "Advanced Algorithms for ACCM, Final Report", ALPHATECH Technical Report, TR-869, ALPHATECH, Inc., February 1998.

[5] C. J. Donohue, O. P. Kreidl, R. Kwon, and J. Birge, "Advanced ISR Management Planning Under Uncertainty: Mathematical Solutions," ALPHATECH Technical Report, TR-903, ALPHATECH, Inc., 1998.

[6] C. T. Lawrence and W. H. Bennett, "Advanced ISR Management, Multi-Asset Synchronizer: Research \& Development Status \& Accomplishments," AIM Technical Report, April 2001.

[7] Bertsimas, D. J. and D. Simchi-Levi, "A New Generation of Vehicle Routing Research: Robust Algorithms, Addressing Uncertainty," Operations Research, 44, 1996, pp. 286-304.

[8] D. P. Bertsekas, Dynamic Programming and Optimal Control, Vols. I-II, Athena Scientific, Belmont, MA 1995.

[9] D. P. Bertsekas and J. N. Tsitsiklis, Neuro-Dynamic Programming, Athena Scientific, Belmont, MA 1996.

[10] D. P. Bertsekas and D. A. Castanon, "The auction algorithm for transportation problems," Annals of Operations Research, 20, 1989, pp. 707-732

[11] J. P. Jumper, "Solving Problems for the Future," Air Force Chief of Staff remarks to the Air Force Association National Symposium, Los Angeles, CA, November 16, 2001.

[12] J. Brandstadt, M. Kozak, P. Redmond, and J. Jones, A UAV Flight and Sensor Simulator for Generating Radar and Infrared Detections as Stimuli for Track and Fusion Processors, AFRL/IFEA Technical Report, May 1999.

[13] Joe P. Hill, W. H. Bennett, H. Landau, "Fusion of GMTI Reports from Multiple Platforms in Clutter", Fusion 2002, (submitted). 